\title{
Development of a SMART-system for a Complex Industrial Object Control based on Metaheuristic Algorithms of Swarm Intelligence
}

\author{
Timur SAMIGULIN $^{1,2}$, Olga ShIRYAYEVA ${ }^{1,3}$ \\ ${ }^{1}$ A. Burkitbaev Institute of Industrial Automation and Digitalization, \\ Satbayev University, \\ 22a Satpaev str., Almaty \\ KAZAKHSTAN \\ ${ }^{2}$ Faculty of Information Technologies, \\ Kazakh-British Technical University, \\ 59 Tole bi str., Almaty \\ KAZAKHSTAN \\ ${ }^{3}$ Institute of Information and Computational Technologies MES RK CS, \\ 28 Shevchenko str., Almaty \\ KAZAKHSTAN
}

\begin{abstract}
The article is devoted to the synthesis of a SMART-system for a complex industrial object control based on metaheuristic optimization algorithms and modern industrial equipment from Honeywell Company. There has been developed software for collecting industrial data, automated tuning of typical controllers of a MIMO industrial object based on such intelligent optimization algorithms as ant colony algorithm, grey wolf optimization, dragonfly algorithm and cuckoo search algorithm. These algorithms are used to minimize the developed new modified quality criteria of a MIMO industrial object. The results are integrated into the Honeywell Experion PKS distributed control system for technological process control in the oil and gas industry using a distillation column for purifying gas from impurities as an example. On the basis of the decoupling procedure, the problem of compensating for the influence of the MIMO system interconnections is solved. The paper substantiates the effectiveness of the implementation of the developed SMART-system for solving the problems of optimal complex technological production control in the oil and gas industry on the example of the real production process of the TengizChevroil enterprise.
\end{abstract}

Key-Words: SMART-system for control, MIMO object, modified quality criteria, metaheuristic algorithms, ant colony optimization, cuckoo search, dragonfly algorithm, gray wolf optimizer, PI-controller

Received: March 10, 2021. Revised: September 20, 2021. Accepted: October 3, 2021. Published: October 25, 2021.

\section{Introduction}

Currently, the world's leading companies for the creation of highly efficient distributed systems for complex technological processes control are engaged in the development and implementation of industrial artificial intelligence in production (IAI) [1]. Significant progress in this area is observed due to the development of the following technologies: Smart Manufacturing, Industrial Big Data Analysis, Digital Twin technology, development of control systems based on metaheuristic optimization algorithms, machine learning methods [2] and statistical data analysis. Industrial artificial intelligence is being successfully implemented in aerospace, metallurgy, oil and gas, and other industries. The intellectualization of oil and gas enterprises is of high economic importance, since one day of plant downtime can lead to multi-million dollar losses.

Bioinspired artificial intelligence algorithms such as genetic algorithms (GA), neural networks, swarm intelligence (RI), etc. have become widespread for solving optimization problems in production. For example, the research [3] presents a new way of optimally wells positioning in oil fields using evolutionary calculations. The paper considers a cellular genetic algorithm for finding an optimal solution. The developed method shows high efficiency and increases the diversity of populations in comparison with the classical genetic algorithm by 1.5 times. The research [4] is devoted to solving a multiparameter task of optimizing the process of hydrocarbon production from a gas condensate reservoir based on metaheuristic algorithms of SI. The paper provides a comparative analysis of the 
effectiveness of the application of GA, the grasshopper optimization algorithm, the grey wolf optimization method (GWO), the moth algorithm (MFO) and the particle swarm optimization (PSO). The best results in finding the optimal solution were shown by the PSO and MFO algorithms. The article [5] considers a modified GA with an ant colony (ACO) algorithm in order to optimize the process of alternate injection of water and gas into an oil reservoir. This process is a complex non-linear task that requires a lot of computational calculations and time. The combination of GA with ACO has shown high efficiency and allows to increase the final volume of oil production. The research [6] is devoted to the optimization of flight routes for unmanned aerial vehicles (UAVs) for a network of oil and gas pipelines based on evolutionary algorithms. A comparative analysis of the GA, the simulated annealing (SA) and the adaptive genetic algorithm for simulated annealing (AGASA) solving the problem of planning flight routes was carried out. The most efficient method based on simulation results is AGASA.

Methods of artificial intelligence (AI) are successfully applied to tune PID controller parameters. For example, in [7], there is considered the synthesis of a PID controller based on stochastic optimization of a deep neural network (Stochastic Optimization of Deep Networks). In article [8], the PID controller parameters are tuned using the metaheuristic Cuckoo Search (CS) algorithm for various engineering applications. In [9], there is proposed a new optimal combined fuzzy PID controller using the Dragonfly Algorithm (DA) for solving the power system control problem.

Nowadays, there have been developed a large number of AI-based optimal systems for onedimensional objects control (SISO), however, most complex industrial objects have a multidimensional, multi-connected structure (MIMO).

For effective work with MIMO objects, it is relevant to develop a SMART-system, which is an information technology for integration with industrial distributed control systems (for example, Honeywell Experion PKS), capable of collecting and intelligent processing of data, process modeling and supervisory control in real time with a high level of adaptation to changing environmental conditions [10]. The SMART system for the Industry 4.0 concept [11] should perform the functions of intelligent monitoring of the enterprise, production management, planning and logistics, from the process of collecting data from sensors to scheduling work shifts and issuing reports. So, for example, in the scientific work [12] there is considered a SMART development of oil fields, to ensure a highly efficient and environmentally friendly process of oil production, through digitalization and intellectualization of control systems.

The introduction of intelligent SMART control systems allows to implement high-tech, reliable, safe and cost-effective production. The application of intelligent approaches is relevant for the oil and gas industry, where one hour of production downtime as a result of an accident or repair carries huge monetary losses.

The following structure of the article is proposed: Section 2 discusses the formulation of the research problem, Section 3 describes the mathematical model of a complex MIMO object of control, Section 4 is devoted to the development of a SMART control system, Section 5 contains the results of modeling and experiments, Section 6 contains information on the development of a software implementation of a SMART-system based on Honeywell equipment, in the conclusion the main conclusions are presented.

\section{Problem Formulation}

The statement of the research problem is formulated as follows: it is necessary to develop a SMARTsystem for a complex multidimensional, multiconnected object control using the example of a real production process at TengizChevroil for purifying gas from impurities in a distillation column (DC) based on metaheuristic algorithms of swarm intelligence and modern industrial equipment from Honeywell.

To create software for finding the optimal control strategy and automated tuning of the parameters of PI controllers of a complex MIMO object. To find the parameters of PI regulators of a complex MIMO object using intelligent algorithms that minimize the developed modified quality criteria taking into account the dynamics of a complex object. To integrate a SMART-system into Honeywell Experion PKS software product for the intellectualization of industrial production.

\section{Mathematical Model of a Complex MIMO-control Object of a Distillation Column}

As an object of the oil and gas industry, there is considered a DC, which is the most common tool for the process of natural gas purification from various impurities and for obtaining the final 
product. The Fig. 1 shows a technological scheme of a gas purification process in a DC, in which the $\mathrm{L}-\mathrm{V}$ structure is presented, where $\mathrm{L}$ is the distillate flow rate and $\mathrm{V}$ is the steam flow rate for controlling distillation with product composition control. The flow $\mathrm{L}$ and vapor $\mathrm{V}$ rates are the control inputs [13]. The task of the controllers is to maintain the desired concentrations of products $\chi_{d}, \chi_{b}$ at the output of the unit.

The DC model can be represented by a system with two inputs and two outputs [14], which corresponds to a MIMO control system of the following form

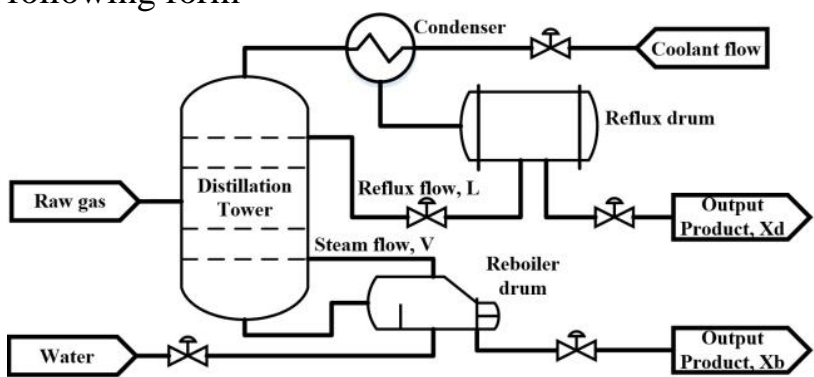

Fig. 1: Technological scheme of a gas purification process

$$
\left\{\begin{array}{l}
\frac{d \chi_{d}(t)}{d t}=-\frac{1}{75 s+1} \chi_{d}(t)+\frac{87.8}{75 s+1} L(t)+\frac{108.2}{75 s+1} V(t) \\
\frac{d \chi_{b}(t)}{d t}=-\frac{1}{75 s+1} \chi_{b}(t)-\frac{109.6}{75 s+1} V(t)-\frac{86.4}{75 s+1} L(t)
\end{array}\right.
$$

where $\chi_{D}-$ distillate concentration; $\chi_{B}-$ concentration of product from the bottom of the column; $\mathrm{L}(\mathrm{t})$ - liquid flow rate (control input for $\chi_{D}$, but affects $\chi_{B}$ along the loop of the relationship); $\mathrm{V}(\mathrm{t})$ - vapor flow rate (control input for $\chi_{B}$, but affects $\chi_{D}$ along the loop of the relationship).

The proposed mathematical model represents the main dynamics of the processes occurring in the DC. Model (1) reflects the inertial dependence of the time variation of several output signals $\chi_{d}, \chi_{b}$ on the time variation of the input control signals, as well as on the influence of interconnections. This defines the system as a MIMO-control object. This model is linear, stationary, continuous in time.

The analysis of the results of the MIMO-object simulation showed that in order to achieve the desired technological requirements, it is necessary to use a control procedure. In accordance with the technological requirements for the process, control is necessary to provide such specified quality ratings as overshoot, rising time, settling time. This process is quite difficult to manage due to the influence of interconnections on the main channels. To solve this problem, in the next section, there is applied the decoupling procedure and is developed a SMART control system [15].

\section{Development of a SMART-system for a Complex MIMO-object Control}

There has been developed a structural scheme of a SMART-system for a complex MIMO object control of a DC (1), which consists of 4 stages (Fig. 2).

Stage 1. Collection and processing of technological data of the DC control object from the Honeywell Experion PKS distributed control system:

- readings from field sensors, generated signals "alarms", "alerts" and "events";

- information about the parameters of the programmable logic controller (PLC). For example, information about the load of the central processor, communication channels, temperature of PLC modules, etc.

Stage 2. Adjustment of PI controllers for a system of a complex MIMO object control based on bioinspired algorithms, which minimize the selected quality criteria and provide the specified quality assessments. For this stage, there have been developed new modified quality criteria, taking into account the relationships between the subsystems of a complex MIMO object control (1). To perform calculations, the Mathwork MATLAB software is used, where a mathematical model of the MIMO control object is assembled in Simulink. Four bioinspired optimization algorithms were selected: ACO [16], GWO [17], DA [18] and CS [19], based on the Mean Absolute Percentage Error (MAPE) assessment. In the course of the research, the results of runs of ten AI algorithms were obtained, most of which did not give satisfactory results in terms of the dynamics of the MIMO system and its quality assessments. Only the algorithms ACO, GWO, DA and CS made it possible to obtain the results of regulator tuning, in which the MIMO control system for a given industrial object remains stable and satisfies the desired quality assessments.

Stage 3. Modeling the process of gas purifying in the DC based on synthesized controllers (obtained at stage 2) in Honeywell Unisim Design software [2021]. Unisim Design is a tool for static and dynamic analysis of technological processes of the oil and gas industry and the chemical industry. 


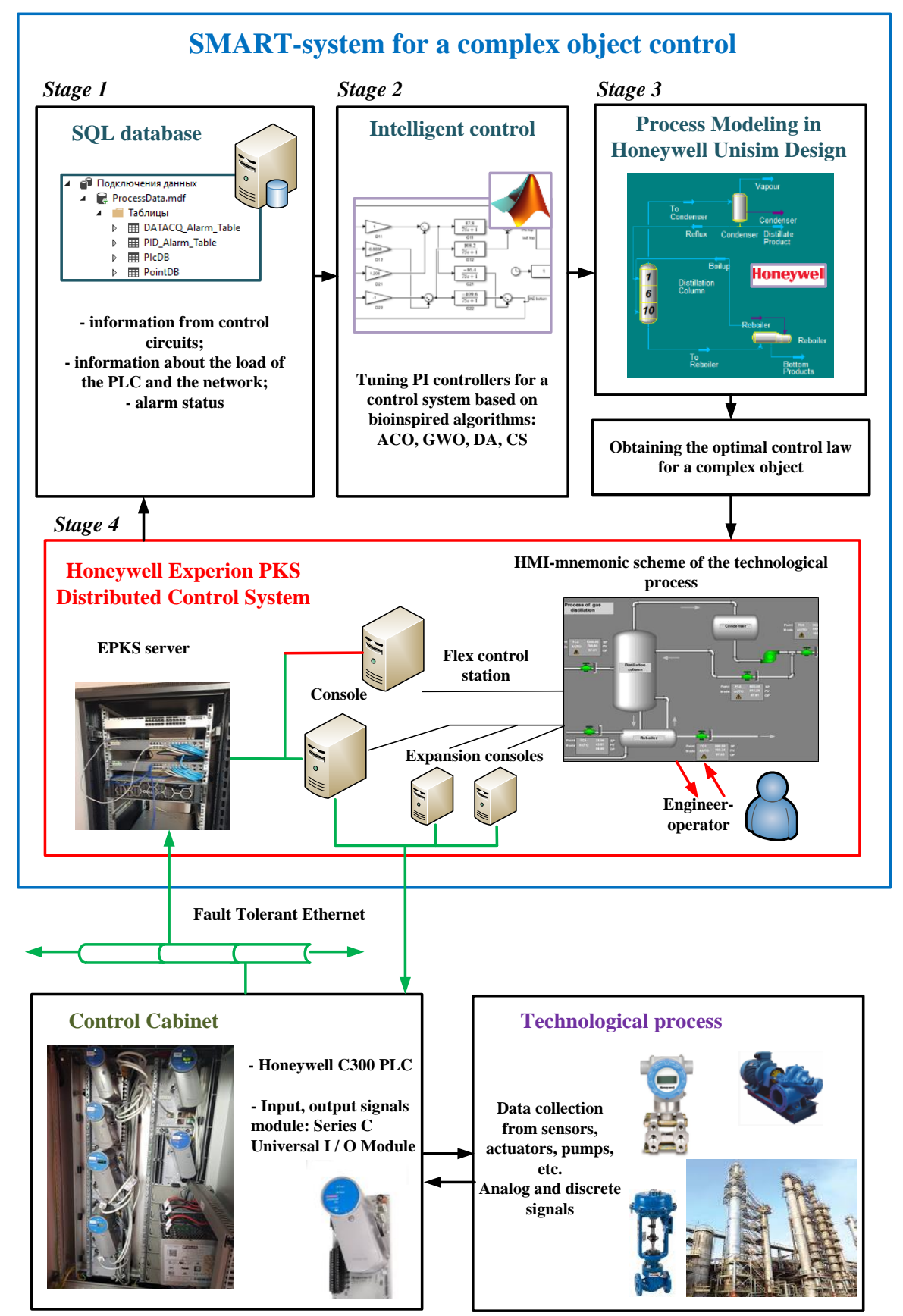

Fig. 2: Structural scheme of a SMART-system for a complex MIMO object control

Stage 4. Software implementation of the control system in Honeywell Experion PKS. To implement a SMART control system in DCS, it is necessary to configure the production model, network topology, configure the Honeywell C300 industrial controller in the Control Builder application and configure the control logic. Then to implement the connection to the control station, develop the HMI-interface of the technological process in the HMI-Web Display Builder program. Finally, it is necessary to set up an emergency notification system and automated reporting.

\subsection{Development of Modified Quality Criteria for a Complex MIMO Object}

In order to implement the 2nd stage of the development of the SMART-system for a complex MIMO object control, it is necessary to develop new modified quality criteria.

In accordance with different requirements for the technological process of a complex MIMO object of the DC (1), there have been developed three modified quality criteria.

1. Modified root mean squared quality criterion ISE_M for two control circuits $L$ and $V$, which is the sum of the integral squared error and the 
criterion limiting the system overshoot within 5\% for each control loop.

$$
\left\{\begin{array}{c}
J_{I S E_{-} L}=\int_{\mathrm{t}=0}^{\mathrm{n}}\left(\hat{y}_{L}-y_{L}\right)^{2} d t+\int_{t=0}^{n} K_{\infty} \cdot \max \left\{0, y_{L}-\hat{y}_{L O}\right\} d t \\
J_{I S E_{-} V}=\int_{\mathrm{t}=0}^{\mathrm{n}}\left(\hat{y}_{V}-y_{V}\right)^{2} d t+\int_{t=0}^{n} K_{\infty} \cdot \max \left\{0, y_{V}-\hat{y}_{V O}\right\} d t \\
J_{I S E \operatorname{cost}}=J_{I S E_{-} L}+J_{I S E_{-} V}
\end{array}\right.
$$

where $J_{I S E, L}, J_{I S E, V}$ - quality criterion for $\mathrm{L}$ and $\mathrm{V}$ circuits respectively, $y_{L}, y_{v}$ - current process value by circuits $\left(\chi_{D}\right.$ and $\chi_{B}$, respectively), $\hat{y}_{L}, \hat{y}_{V}-$ corresponding desired variable values, $\hat{y}_{L O}, \hat{y}_{V O}$ allowable overshoot levels, $K_{\infty}$ - infinitely high amplification factor, $J_{I S E \text { cost }}$ - general quality criterion for a MIMO object of a DC, $t=0, \ldots, n-$ technological process modeling time.

2. Modified Integral Time Absolute Error quality criterion ITAE_M for two circuits L and V:

$$
\left\{\begin{array}{c}
J_{I T S E_{-} L}=\int_{\mathrm{t}=0}^{\mathrm{n}} t\left(\hat{y}_{L}-y_{L}\right)^{2} d t+\int_{t=0}^{n} K_{\infty} \cdot \max \left\{0, y_{L}-\hat{y}_{L O}\right\} d t \\
J_{\text {ITSE_V }}=\int_{\mathrm{t}=0}^{\mathrm{n}} t\left(\hat{y}_{V}-y_{V}\right)^{2} d t+\int_{t=0}^{n} K_{\infty} \cdot \max \left\{0, y_{V}-\hat{y}_{V O}\right\} d t \\
J_{\text {ITSE } \operatorname{cost}}=J_{\text {ITSE }}+J_{\text {ITSE_V }}
\end{array}\right.
$$

where $J_{I T S E, L}, J_{I T S E, V}$ - quality criterion for $\mathrm{L}$ and $\mathrm{V}$ circuits respectively, $J_{\text {ITSE cost }}$ - general quality criterion for a MIMO object of a DC.

3. Modified Integral Absolute Error quality criterion IAE_M for two circuits L and V:

$$
\left\{\begin{array}{c}
J_{I A E_{-} L}=\int_{\mathrm{t}=0}^{n}\left|\hat{y}_{L}-y_{L}\right| d t+\int_{t=0}^{n} K_{\infty} \cdot \max \left\{0, y_{L}-\hat{y}_{L O}\right\} d t \\
J_{I A E_{-} V}=\int_{\mathrm{t}=0}^{n}\left|\hat{y}_{V}-y_{V}\right| d t+\int_{t=0}^{n} K_{\infty} \cdot \max \left\{0, y_{V}-\hat{y}_{V O}\right\} d t \\
J_{I A E \text { cost }}=J_{\text {IAE } L}+J_{\text {IAE } V}
\end{array}\right.
$$

where $J_{I A E, L}, J_{I A E, V}$ - quality criterion for $\mathrm{L}$ and $\mathrm{V}$ circuits respectively, $J_{I A E}$ cost - general quality criterion for a MIMO object of a DC.

The advantage of the developed criteria, in contrast to the existing ones, is as follows: the developed modified quality criteria (2-4) allow taking into account the relationships between the subsystems of a complex MIMO object of the DC (1) and limit the system overshoot to 5\%.

\subsection{Synthesis of Intelligent PI Controllers}

In order to develop a SMART-system for controlling the gas purification process in a DC (1), it is necessary to synthesize PI controllers for two circuits of the system $\mathrm{L}$ and $\mathrm{V}$, in order to ensure a minimum of modified quality criteria (2-4) based on the optimization algorithms ACO, GWO, DA, $\mathrm{CS}$ in the following form:

$$
\begin{aligned}
& u_{L}(t)=K_{p_{L}} e_{L}(t)+\int_{0}^{t} K_{i L} e_{L}(t) d(t), \\
& u_{V}(t)=K_{p V} e_{V}(t)+\int_{0}^{t} K_{i V} e_{V}(t) d(t),
\end{aligned}
$$

where $K_{p_{L}}, K_{i L}, K_{p V}, K_{i V}$ - the coefficients of the proportional and integral components of the controllers along the corresponding circuits $\mathrm{L}$ and $\mathrm{V}, e_{L}(\mathrm{t}), e_{V}(t)-$ mismatch errors corresponding to the circuits between the setpoint and the reading from the sensors, $u_{L}(t), u_{V}(t)-$ control signals of the $\mathrm{L}$ and $\mathrm{V}$ circuits.

The Fig. 3 shows a structural scheme of the PI controllers synthesis process, where $G_{11}, G_{12}, G_{21}, G_{22}$ - the transfer functions of the model (1), $u_{\mathrm{L}}$ and $u_{\mathrm{V}}-$ input control signals, $y_{L}$ and $y_{V}-$ output signals of the control object. Elements $G_{12}, G_{21}$ are cross-links indicating the mutual influence of the first input on the second one and vice versa.

In order to get rid of the influence of cross-links when creating a control system with two controllers, a decoupling control procedure is carried out. for system (1), the coefficients of the decoupling matrix are presented as follows:

$$
D=\left[\begin{array}{ll}
D_{11} & D_{12} \\
D_{21} & D_{22}
\end{array}\right]=\left[\begin{array}{cc}
1 & -0.8038 \\
1.206 & -1
\end{array}\right]
$$

Intelligent tuning of the parameters of PI controllers for control circuits is considered as a multiparameter optimization problem [22]. To solve the criterion minimization problem (2-4), four A algorithms are selected that provide the system with the desired dynamics: ACO, GWO, DA and CS. The configurations of these algorithms are presented in detail in [16-19].

As an initial population of AI algorithms, there are formed the initial parameters of PI controllers (5), which satisfy the stability condition for a complex MIMO object of the DC (1) with a decoupling matrix (6).

General settings for optimization algorithms: the number of search variables -4 , the number of iterations - about 100, the lower bounds for variables -0.01 , the upper bounds for variables 30.

Initial parameters: 


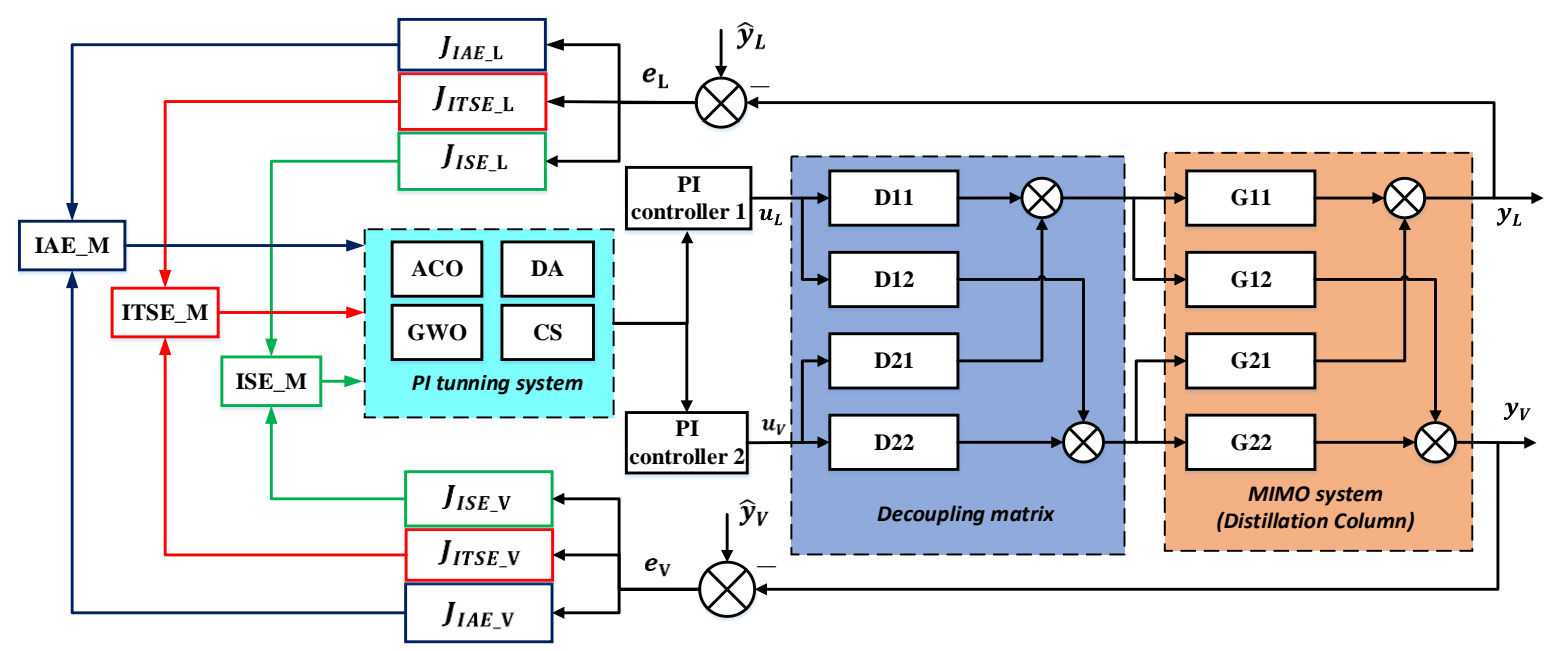

Fig. 3: Structural scheme of intelligent tuning of the PI controller

a) ACO: the number of ants - 400, the alpha coefficient -0.8 , the beta coefficient -0.2 , the evaporation rate coefficient -0.7 .

b) GWO: the number of wolves -100 .

c) CS: the number of nests -25 , the speed of detection of eggs by the owner -0.25 , tolerance $1.0 \mathrm{e}-5$

d) DA: the maximum number of generations 100 , the number of search agents -25 units.

Table 1 presents the results of calculating the controllers coefficients (5).

Table 1. Calculated coefficients of PI-controllers

\begin{tabular}{|l|l|c|c|c|c|}
\hline \multirow{2}{*}{$\begin{array}{l}\text { Algo- } \\
\text { rithm }\end{array}$} & \multirow{2}{*}{$\begin{array}{l}\text { Quality } \\
\text { criterion }\end{array}$} & \multicolumn{2}{|c|}{ Control loop, L } & \multicolumn{2}{c|}{ Control loop, V } \\
\cline { 3 - 6 } & $K_{p_{L}}$ & $K_{i L}$ & $K_{p V}$ & $K_{i V}$ \\
\hline \multirow{4}{*}{ ACO } & IAE-M & 5,6876 & 0,1249 & 21,614 & 0,0100 \\
\cline { 2 - 6 } & ITSE-M & 3,2522 & 0,0459 & 14,511 & 0,1209 \\
\cline { 2 - 6 } & ISE-M & 5,7656 & 0,0279 & 26,391 & 0,0399 \\
\hline \multirow{4}{*}{ GWO } & IAE-M & 11,4935 & 0,3352 & 30,000 & 0,3239 \\
\cline { 2 - 6 } & ITSE-M & 7,1921 & 0,1373 & 30,000 & 2.2926 \\
\cline { 2 - 6 } & ISE-M & 4,8406 & 0,1030 & 29,5761 & 0,5759 \\
\hline \multirow{4}{*}{ DA } & IAE-M & 1,2951 & 0,1201 & 6,3392 & 0,0100 \\
\cline { 2 - 6 } & ITSE-M & 20,8673 & 0,2785 & 25,7686 & 16,059 \\
\cline { 2 - 6 } & ISE-M & 19,6164 & 0,5127 & 29,3209 & 21,306 \\
\hline \multirow{5}{*}{ CS } & IAE-M & 4,8060 & 0,100 & 29,9472 & 0,1000 \\
\cline { 2 - 6 } & ITSE-M & 4,9463 & 0,1078 & 30,0000 & 0,2918 \\
\cline { 2 - 6 } & ISE-M & 4,9012 & 0,0811 & 30,0000 & 0,0100 \\
\hline
\end{tabular}

In accordance with the theory of automatic control, the best regulator is selected in the course of comparative analysis of assessments of the transient processes quality. For the transient processes of two loops of the MIMO object, there were obtained quality assessments (Table 2), which correspond to the overclocking part of the transient. For a given industrial object, the desired quality assessment is an overshoot in two control loops of no more than $5 \%$. As a result of the analysis of transient
The following simulation equipment was used:

1. Personal computer characteristics: Intel Core i7 4770k 3.5 GHz, RAM DDR3 16GB, Nvidia GeForce GTX970, 512GB SSD, OS Windows 10.

Server characteristics Dell Power Edge R740: 20 CPUs $x$ Intel ${ }^{\circledR}$ Xeon ${ }^{\circledR}$ Silver $4114(2.2 \mathrm{GHz})$, Memory 128GB, Storage 2 TB, VMWare ESXi 6.7. After calculating the parameters of PI controllers, the most appropriate control law is selected, depending on the following parameters: overshoot, rising time, settling time.

\section{Results of Modeling and Experiments. Discussion Part.}

There was developed software in MATLAB environment for parallel synthesis of PI controllers (5) and for the selection of the best algorithm. The Fig. 4 shows the transient characteristics for the control circuits $\mathrm{L}, \mathrm{V}$ with decoupling elements and PI controllers, tuned using ACO, DA, CS, GWO, respectively (Table 1 ).

In order to implement the ACO and DA algorithms, 100 iterations are not enough to find the optimal solution for a given quality criterion (Fig. 4, (a), (b), (e), (f)). For the GWA and CS algorithms, 80 iterations were enough (Fig. 4, (c), (d), (g), (h) processes, there were obtained other quality assessments: rise time, settling time (Table 2).

In the course of a comparative analysis of quality assessments for two control loops (Table 2), the following results were obtained.

As part of the discussion part, we present the results of a comparative analysis of the use of intelligent optimization algorithms in order to provide the desired quality ratings. In accordance with the specified overshoot requirements, the CS algorithm has the best performance. The average 
overshoot rate for the two loops is the smallest and does not exceed $5 \%$ while minimizing any quality criterion.

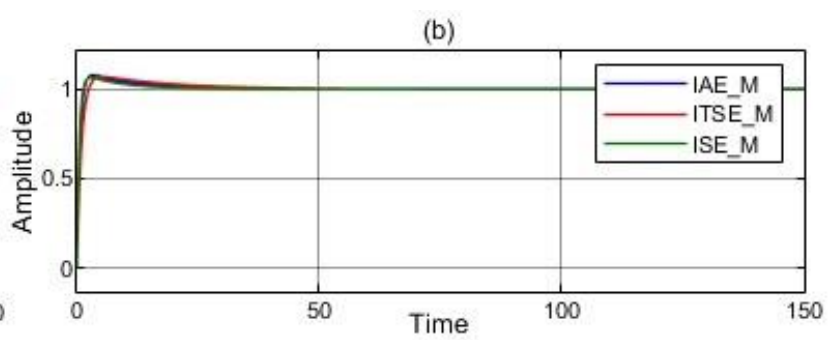

(d)

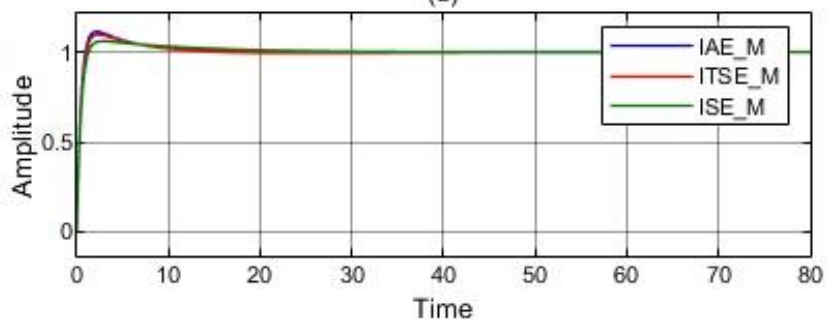

(f)
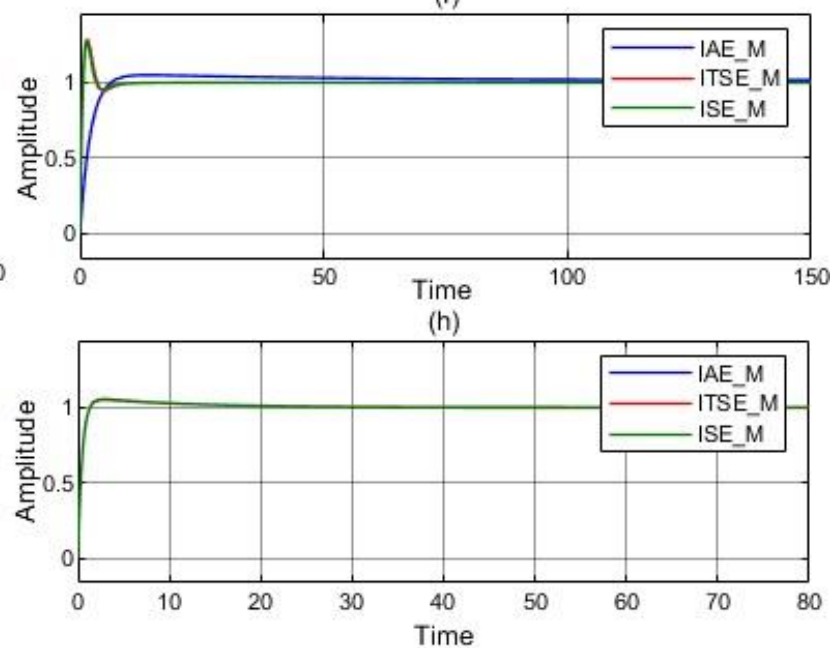

Fig. 4: Step responses of the distillation process. (a) ACO-PI controller 1. (b) ACO-PI controller 2. (c) GWO-PI controller 1. (d) GWO-PI controller 2. (e) DA-PI controller 1. (f) DA-PI controller 2. (g) CS-PI controller 1. (h) CS-PI controller 2

Also, overshoot in two control loops (Table 1) does not exceed 5\% when using the DA algorithm to minimize one modified ISE_M quality criterion.

In accordance with the comparative analysis of transient processes and quality assessments, it is possible to draw conclusions, including on the speed of the system: the use of the ACO and GWO algorithms provided the system, on average over two loops, with a better rise time and regulation time than the CS and DA algorithms. However, the desired technological requirement for the system was overshoot, therefore, these algorithms do not meet the quality requirements of the object under consideration.

This is due to the fact that, according to the technological regulations, restrictions on the size of the maximum overshoot along the $\mathrm{L}$ and $\mathrm{V}$ circuits are imposed on the control object of the distillation column to ensure a good working condition, where

exceeding this limit can lead to an accident. With other specified quality ratings, these algorithms can provide an efficient mode of operation.

To confirm the effectiveness of the chosen metaheuristic algorithm, there was performed a statistical analysis based on the Mean Absolute Percentage Error (MAPE) assessment, which is presented in Table 3 [23]. For each control loop (L and $\mathrm{V}$ ), the parameter is calculated for the modified criteria IAE_M, ITSE_M and ISE_M.

The advantage of the conducted research and their innovative future lies in the fact that as a result of a comparative analysis of various intelligent optimization algorithms, the problem of synthesizing a MIMO system using new developed 
modified quality criteria and integration into a modern distributed control system Honeywell Experion PKS was successfully solved.

Table 2. Algorithm performance for two control loops

\begin{tabular}{|c|c|c|c|c|}
\hline $\begin{array}{l}\text { Algo- } \\
\text { rithm }\end{array}$ & $\begin{array}{l}\text { Quality } \\
\text { criterion }\end{array}$ & $\begin{array}{l}\text { Overshoo } \\
\text { t, } \%\end{array}$ & $\begin{array}{l}\text { Rise } \\
\text { time, } \mathrm{s}\end{array}$ & $\begin{array}{l}\text { Settling } \\
\text { time, s }\end{array}$ \\
\hline \multicolumn{5}{|c|}{ Control loop L with PI controller 1} \\
\hline \multirow[t]{3}{*}{$\mathrm{ACO}$} & ISE-M & 4.994 & 7.499 & 46.017 \\
\hline & ITSE-M & 0.354 & 9.859 & 39.479 \\
\hline & IAE-M & 5.077 & 7.469 & 45.684 \\
\hline \multirow[t]{3}{*}{ GWO } & ISE-M & 5.866 & 7.494 & 42.387 \\
\hline & ITSE-M & 2.807 & 9.078 & 41.393 \\
\hline & IAE-M & 6.003 & 8.389 & 30.526 \\
\hline \multirow[t]{3}{*}{ DA } & ISE-M & 0.065 & 17.531 & 65.906 \\
\hline & ITSE-M & 0.002 & 13.243 & 36.065 \\
\hline & IAE-M & 3.115 & 12.890 & 43.176 \\
\hline \multirow[t]{3}{*}{$\mathrm{CS}$} & ISE-M & 1.610 & 47.572 & 73.318 \\
\hline & ITSE-M & 1.886 & 47.461 & 72.946 \\
\hline & IAE-M & 0.536 & 47.814 & 74.056 \\
\hline \multicolumn{5}{|c|}{ Control loop V with PI controller 2} \\
\hline \multirow[t]{3}{*}{$\mathrm{ACO}$} & ISE-M & 7.533 & 8.667 & 29.207 \\
\hline & ITSE-M & 7.020 & 10.532 & 32.244 \\
\hline & IAE-M & 6.652 & 7.976 & 28.499 \\
\hline \multirow[t]{3}{*}{ GWO } & ISE-M & 11.312 & 7.453 & 28.046 \\
\hline & ITSE-M & 9.636 & 7.770 & 27.877 \\
\hline & IAE-M & 4.914 & 10.034 & 50.357 \\
\hline \multirow[t]{3}{*}{$\mathrm{DA}$} & ISE-M & 3.324 & 19.924 & 44.975 \\
\hline & ITSE-M & 28.569 & 6.087 & 33.507 \\
\hline & IAE-M & 27.560 & 5.792 & 32.714 \\
\hline \multirow[t]{3}{*}{$\mathrm{CS}$} & ISE-M & 2.055 & 49.234 & 66.725 \\
\hline & ITSE-M & 2.869 & 49.192 & 66.995 \\
\hline & IAE-M & 2.472 & 49.247 & 66.404 \\
\hline
\end{tabular}

Table 3. Statistical assessment of the efficiency of algorithms for modified quality criteria

\begin{tabular}{|c|c|c|c|c|c|c|}
\hline \multicolumn{7}{|c|}{ Mean Absolute Percentage Error (MAPE, \%) } \\
\hline \multirow{2}{*}{$\begin{array}{l}\text { Qua- } \\
\text { lity }\end{array}$} & \multicolumn{2}{|c|}{ IAE-M } & \multicolumn{2}{|c|}{ ITSE-M } & \multicolumn{2}{|c|}{ ISE-M } \\
\hline & $\begin{array}{l}\Omega \\
0 \\
0 \\
0 \\
0 \\
0 \\
0 \\
0\end{array}$ & $\begin{array}{l}0 \\
0 \\
0 \\
0 \\
8 \\
8 \\
< \\
<\end{array}$ & $\begin{array}{l}0 \\
8 \\
0 \\
0 \\
8 \\
0 \\
0\end{array}$ & 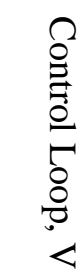 & $\begin{array}{l}\hat{8} \\
8 \\
0 \\
8 \\
8 \\
0 \\
0\end{array}$ & $\begin{array}{l}\delta \\
8 \\
0 \\
0 \\
0 \\
8 \\
8\end{array}$ \\
\hline $\mathrm{ACO}$ & 0.14 & 0.42 & 0.16 & 0.05 & 4.11 & 0.25 \\
\hline GWO & 0.12 & 0.01 & 0.39 & 0.01 & 0.63 & 0.02 \\
\hline DA & 0.86 & 1.72 & 0.01 & 0.01 & 0.09 & 0.01 \\
\hline CS & 0.65 & 0.16 & 0.60 & 0.02 & 0.51 & 0.34 \\
\hline
\end{tabular}

Based on the results of statistical analysis, it can be concluded that the metaheuristic algorithms described in the study showed results that meet the requirements for the accuracy of calculations.

\section{Software Implementation of a SMART Control System based on Honeywell Equipment}

A SMART control system is implemented on the basis of the "Honeywell" laboratory of the KazakhBritish Technical University (Almaty, Kazakhstan). An Experion PKS Distributed Control System (DCS) with a Dell EMC R740 server is used as an automation platform.

There has been developed the structure of the database and software in the C\# language to collect information on the operation of the distillation column of the TengizChevroil enterprise, which allows automated data collection in Microsoft SQL. Information about the current equipment status and control circuits parameters are generated by the Experion PKS DCS into Microsoft Excel reports for further intelligent analysis. The SMART control system is implemented for the Honeywell C300 programmable logic controller.

On the basis of Experion PKS DCS there were developed (Fig. 5): control strategy for Honeywell C300 controller based on synthesized intelligent controllers (Section 4, Stage 2); HMI-interface of the MIMO object of the DC was created for visualization of the technological process; Alarm systems were configured for HMI mnemonic schemes and Honeywell control station.

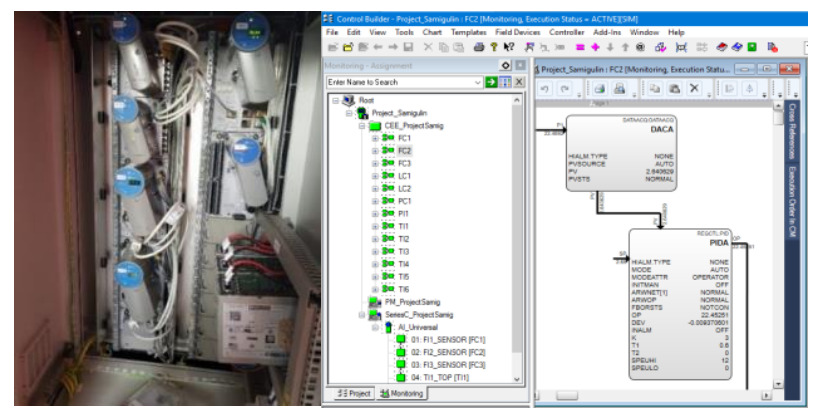

Fig. 5: Implementation of a control strategy on the C300 controller

The developed mnemonic scheme of a complex MIMO object allows operators to monitor the state of the technological process in real time. That is, a real-time system is understood as such a control system that reacts to influences and perturbations exerted on the control object in strict time frames 
necessary for adequate process control [24]. With the help of the developed mnemonic diagram, the operator traces the dynamics of the control object using trends and monitors the absence of an alarm that occurs when abnormal situations appear. Also, the engineer has the ability to trace the chain of emerging alarms to determine the source of an industrial accident.

The Fig. 6 shows the operator display running on the Flex Station.

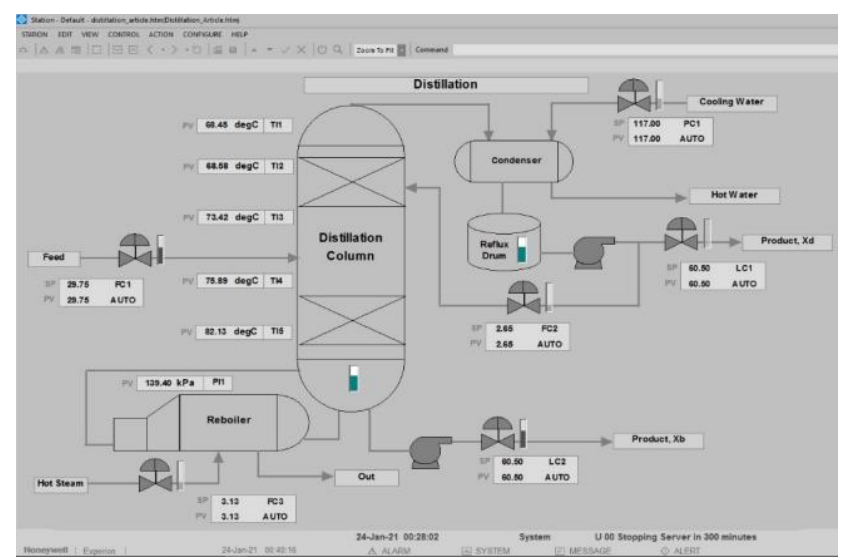

Fig. 6: DC mnemonic scheme in Experion PKS

When developing control logic based on Honeywell equipment, it is necessary to take into account the limitations imposed on the Experion PKS distributed control system, such as the maximum number of process points stored in the database $-45,000$ for a single server. Limitation for Honeywell C300 controller: the maximum number of Input / Output modules for one controller is 64; base execution periods - $20 \mathrm{~ms}$, memory size - 16 $\mathrm{MB}$, maximum number of objects in control logic 4095.

The developed SMART control system, as well as software implementation on the Experion PKS platform, can be used to control real technological processes at TengizChevroil enterprise.

\section{Conclusion}

The paper presents a developed SMART-system for a complex MIMO object control of TengizChevroil based on metaheuristic algorithms of swarm intelligence and industrial equipment from Honeywell.

There were developed modified quality criteria for a complex MIMO-system, taking into account the influence of interconnections and the dynamics of the technological process. A model has been developed for tuning the parameters of PI controllers of a complex MIMO system based on metaheuristic algorithms (ACO, GWO, DA, CS) for modified quality criteria that provide the desired dynamics and stability of the system. An analysis of the effectiveness of artificial intelligence algorithms used in the study is carried out, where the CS algorithm was singled out as the most suitable for solving the problem. The obtained results are integrated into the distillation column control system implemented in the DCS Experion PKS software product based on the Honeywell C300 controller.

The limitations of using research data for other objects are the dimension of MIMO-objects and their modeling time. In the future, it is planned to develop this method for objects of higher dimension.

The authors are grateful to the trainer André Bos from Honeywell NL Automation College, Amsterdam, The Netherlands for providing training on the Experion PKS distributed control system.

\section{References:}

[1] Lee, J., Davari, H., Singh, J., Pandhare, V., Industrial Artificial Intelligence for industry 4.0-based manufacturing systems, Manufacturing Letters, Vol.18, 2018, pp. 2023.

[2] Guillen, D.P, Machine Learning Applications in Advanced Manufacturing Processes, JOM 72, 2020, pp. 3906-3907.

[3] Cunha A.A.L., Duncan G., Bontempo A., Pacheco M.A.C., Optimum Wells Placement in Oil Fields Using Cellular Genetic Algorithms and Space Efficient Chromosomes, Intelligent Systems and Applications. IntelliSys 2016. Studies in Computational Intelligence, Vol. 751, 2018, pp. 15-38.

[4] Janiga, D., Czarnota, R., Stopa, J. et al, Utilization of nature-inspired algorithms for gas condensate reservoir optimization, Soft Computing, Vol. 23, 2019, pp. 5619-5631.

[5] Nait Amar, M., Zeraibi, N. \& Redouane, K., Optimization of WAG Process Using Dynamic Proxy, Genetic Algorithm and Ant Colony Optimization, Arabian Journal for Science and Engineering, Vol. 43, 2018, pp. 6399-6412.

[6] Yan, Y., Liang, Y., Zhang, H. et al., A twostage optimization method for unmanned aerial vehicle inspection of an oil and gas pipeline network, Petroleum Science, Vol. 16, 2019, pp. 458-468.

[7] An, W., Wang, H., Sun, Q., Xu, J., Dai, Q., Zhang, L., A PID controller approach for stochastic optimization of deep networks, 
IEEE/CVF Conference on Computer Vision and Pattern Recognition, 2018, pp. 8522-8531.

[8] Sethi R., Panda S., Sahoo B.P., Cuckoo Search Algorithm Based Optimal Tuning of PID Structured TCSC Controller, Computational Intelligence in Data Mining - Volume 1. Smart Innovation, Systems and Technologies, Vol. 31, 2015, pp. 251-263.

[9] Kouba, N., Menaa, M., Hasni, M., Boudour, M., A novel optimal combined fuzzy PID controller employing dragonfly algorithm for solving automatic generation control problem. Electric Power Components and Systems, Vol. 46, No. 19-20, 2018, pp. 2054-2070.

[10] Samigulina, G.A., Samigulina, Z.I., Development of Smart technology for complex objects prediction and control on the basis of a distributed control system and an artificial immune systems approach, Advances in Science. Technology and Engineering Systems Journal (ASTESJ), Vol. 4, No.3, 2019, pp. 7587.

[11] Zheng, P., Wang, H., Sang, Z. et al., Smart manufacturing systems for Industry 4.0: Conceptual framework, scenarios, and future perspectives. Frontiers in Mechanical Engineering, Vol. 13, 2018, pp. 137-150.

[12] Liu, H., Ma, X., Smart oilfield development and transition of petroleum-based cities. Frontiers of Engineering Management. Vol. 6, 2019, pp. 299-301.

[13] Skogestad, S., Morari, M., LV-control of a high-purity distillation column, IFAC Proceeding Volumes, Vol. 20, No.5, 1987, pp. 387-396.

[14] Skogestad, S., Postlethwaite, I, Multivariable Feedback Control: Analysis and Design. John Wiley \& Sons, Inc., 2001.

[15] Liu, L., Tian, S., Xue, D. et al., A Review of Industrial MIMO Decoupling Control, International Journal of Control, Automation and Systems, Vol. 17, 2019, pp.1246-1254.

[16] Chiha, I., Liouane, N., Borne, P., Tuning PID Controller Using Multi objective Ant Colony Optimization, Applied Computational Intelligence and Soft Computing, 2012.

[17] Rezaei H., Bozorg-Haddad O., Chu X., Grey Wolf Optimization (GWO) Algorithm Advanced Optimization by Nature-Inspired Algorithms, Studies in Computational Intelligence, Vol. 720, 2018, pp. 81-91.

[18] Rahman, C.M., Rashid, T.A.: Dragonfly algorithm and its applications in applied science survey, Computational Intelligence and Neuroscience. 2019.

[19] Ouaarab A., Yang XS.: Cuckoo Search: From Cuckoo Reproduction Strategy to Combinatorial Optimization, Nature-Inspired Computation in Engineering. Studies in Computational Intelligence, Vol. 637, 2016, pp. 91-110.

[20] Patrascioiu, C., Jamali, M., Crude distillation process simulation using Unisim Design simulator, International Journal of Chemical, Materials and Biomolecular Sciences, Vol.12, No.7, 2018, pp. 340-346.

[21] Ramli, N.M., Distillation column. PID control for industrial processes. IntechOpen, 2018.

[22] Nisi, K., Nagaraj, B., Agalya, A., Tuning of a PID controller using evolutionary multi objective optimization methodologies and application to the pulp and paper industry, International Journal of Machine Learning and Cybernetics, Vol. 10, 2019, pp. 2015-2025.

[23] Sumathi, S., Kumar, A. L., Computational Intelligence Paradigms for Optimization Problems Using MATLAB ${ }^{\circledR} /$ SIMULINK ${ }^{\circledR}$, CRC Press, 2015.

[24] Kopetz, H., Real-Time Systems, Springer NY, 2011.

\section{Contribution of Individual Authors to the Creation of a Scientific Article (Ghostwriting Policy)}

Timur Samigulin carried out the conceptualization, the simulation and the optimization.

Olga Ivanovna was responsible for analysis of the case study.

\section{Sources of Funding for Research Presented in a Scientific Article or Scientific Article Itself}

This research has been funded by the Science Committee of the Ministry of Education and Science of the Republic Kazakhstan (Grant No. AP09258508).

\section{Creative Commons Attribution License 4.0 (Attribution 4.0 International, CC BY 4.0)}

This article is published under the terms of the Creative Commons Attribution License 4.0 https://creativecommons.org/licenses/by/4.0/deed.en US 